\title{
Circuit Modeling of Discontinuous Planar Waveguides at High Frequencies
}

\author{
Alessandro Massaro*, Roberto Cingolani, Adriana Passaseo, and Massimo De Vittorio \\ National Nanotechnology Laboratory of CNR-INFM, Distretto Tecnologico-ISUFI, \\ Università del Salento, Via Arnesano, 73100 Lecce, Italy
}

\begin{abstract}
We present an accurate theoretical approach in view to design discontinuous optical waveguides at high frequencies. The model is based on the Floquet's theory and on the variational equivalent circuit. The distributed circuit model is developed through the use of the microwave network theory and the optical theory of the step discontinuities. This accurate approach considers dielectric periodic structures with high dielectric contrast and the transmission line model applied to guided modes of a discontinuous slab waveguide.
\end{abstract}

Keywords: Optical Waveguide, Optical Grating, Integrated Optic.

\section{INTRODUCTION}

Many scientific and engineering applications $s^{1-11}$ can be realized by utilizing dielectric periodic devices realized artificially by introducing defect states in the basic periodic structures. Technological improvements in high frequency periodic structures have led to the fabrication of photonic crystal structures for power dividers, filters, ${ }^{4-9}$ directional couplers, and wavelength division multiplexer WDM. ${ }^{10,11}$ New classes of dielectric materials which are often artificially fabricated for this purpose are referred as photonic crystals and often are characterized by a high dielectric contrast. The effect of the high dielectric contrast, at high working frequencies, is relevant in the frequency response especially in discontinuous structures such as cascade of step discontinuities. Step discontinuities in planar dielectric waveguides are commonly used in integrated circuits ranging from sub-millimetre to optical frequencies. The step discontinuity in dielectric slab waveguides is, in fact, a basic one for several components such as distributed feedback lasers, gratings, transformers, antenna feed, and others. It is thus important to have accurate and reliable theoretical prediction of the behaviour of this discontinuity. Step discontinuity model, presented in this work, starts from the junction between two slabs of different thickness as two lines with different characteristic impedances. As a refinement of this model we introduce the variational equivalent circuit $^{12}$ which minimizes the effect of the discontinuity for a periodic structure. By modeling

\footnotetext{
*Author to whom correspondence should be addressed.
}

a periodic dielectric structure as a cascade of unit cell elements, ${ }^{12-17}$ the error in the evaluation of the frequency response on a single cell increases drastically for a long structure. In this way it is necessary to introduce the transformation ratio which takes into account the amplitude difference of the traveling signal along the discontinuous waveguide. This aspect is considered in the proposed theoretical approach with the equivalent variational circuit of a step discontinuity ${ }^{12}$ and with the passband Floquet's condition. ${ }^{13-15}$ The variational equivalent circuit permits to evaluate the grating transmittivity response with a good accuracy in comparison with the simplified transmission lines circuits presented in previous works. ${ }^{15}$ In this work we present an accurate theoretical design of a GaAs periodic asymmetrical waveguide including the effect of a defect region in the frequency response. Moreover the 3D The Finite Element Method FEM modeling verifies the theoretical transmittivity for a waveguide with five step of discontinuities.

\section{MODAL TRANSMISSION LINE MODELING AND EQUIVALENT VARIATIONAL CIRCUIT}

Periodic air holes in dielectric material can be modeled by the analogy with the cascade step discontinuities (see Fig. 1(a)). Each step discontinuity is a dielectric junction in planar waveguide. This junction considers the core thicknesses $d$ and $D$ of two waveguide parts with length $L_{1}$ and $L_{2}$ respectively (the grating period is $\Lambda=L_{1}+L_{2}$ ). Each junction (unit cell of Fig. 1(b)) is represented by a 


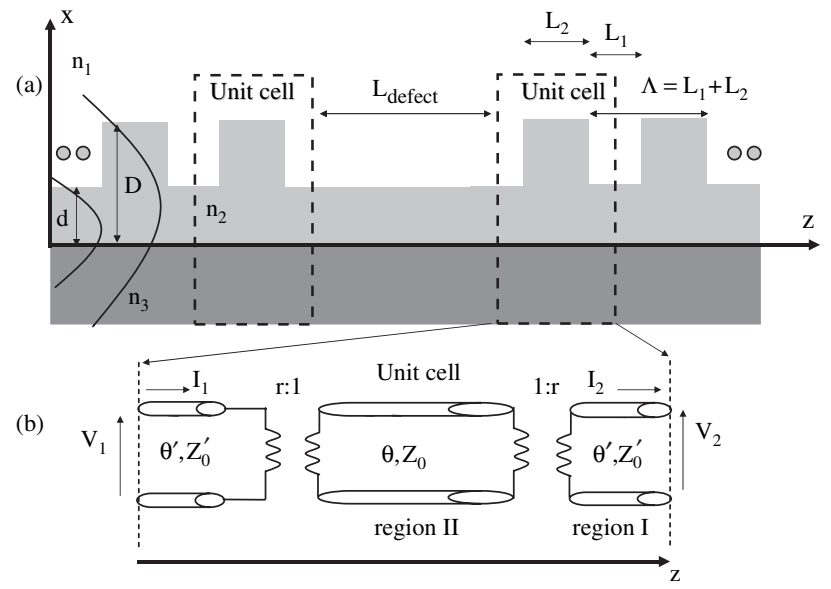

Fig. 1. (a) Asymmetrical slab waveguide with step discontinuities and central defect. (b) Variation equivalent circuit of a single step discontinuity.

variational equivalent circuit: the fundamental modes propagate in the slab waveguide characterized by the electrical length $\theta=\beta_{z} L_{1} / 2$ (region I of Fig. 1(b) with core thickness $d$ ) and in the slab waveguide characterized by the electrical length $\theta^{\prime}=\beta_{z}^{\prime} L_{2}$ (region II of Fig. 1(b)) with core thickness $D$ ), where $\beta_{z}$ and $\beta_{z}^{\prime}$ are the modal propagation constant along the $z$-propagating direction. The transformation ratio $r$ takes into account the effect of the electric field discontinuity and the high dielectric contrast. If more modes are involved, the generic variational equivalent circuit will consider a transmission line for each propagating mode. For the transverse electric TE mode excitation with no variation along the $y$-direction, the generic electric field in regions I and II can be written respectively as

$$
\begin{aligned}
& E_{y}(x, z) \\
& \quad=\left\{\sum_{k} a_{k} \psi_{k}(x)+\int_{0}^{\infty} b\left(k_{x}\right) \phi\left(x ; k_{x}\right) d k_{x}\right\} e^{j\left(\omega t-\beta_{z} z\right)} \\
& E_{y}^{\prime}(x, z) \\
& \quad=\left\{\sum_{k^{\prime}} a_{k^{\prime}}^{\prime} \psi_{k^{\prime}}^{\prime}(x)+\int_{0}^{\infty} b^{\prime}\left(k_{x}^{\prime}\right) \phi^{\prime}\left(x ; k_{x}^{\prime}\right) d k_{x}^{\prime}\right\} e^{j\left(\omega t-\beta_{z}^{\prime} z\right)}
\end{aligned}
$$

where $\psi_{k}$ and $\psi_{k^{\prime}}^{\prime}$ are the guided TE waves, $\phi$ and $\phi^{\prime}$ are the mode functions pertaining to the continuous spectrum, $a_{k}$, and $a_{k^{\prime}}^{\prime}$ are the amplitudes of the guided modes, $b\left(k_{x}\right)$ and $b^{\prime}\left(k_{x}^{\prime}\right)$ are the amplitudes of the continuum modes, $k_{x}$ and $k_{x}^{\prime}$ are the propagation constants along the $x$-direction, finally $\beta_{z}$ and $\beta_{z}^{\prime}$ are the propagation constants along the $z$-propagating direction. By considering the fundamental TE modes the ratio transformation $r$ of Figure 1(b) is defined as ${ }^{12}$

$$
r=\frac{\int_{-\infty}^{+\infty} E_{y}^{*}(x) \sum_{k^{\prime}} a_{k^{\prime}}^{\prime} \psi_{k^{\prime}}^{\prime}(x) d x}{\int_{-\infty}^{+\infty} E_{y}^{*}(x) \sum_{k} a_{k} \psi_{k}(x) d x}
$$

where $E_{y}^{*}(x)$ is the electric field on the junction. In the region I the TE modal profile is given by: ${ }^{12}$

$\psi(x)$

$$
=\left\{\begin{array}{rr}
a e^{-p(x-d) \quad x \geq d} & \\
a \frac{\cos \left(k_{x} x-\bar{\varphi}\right)}{\cos \left(k_{x} d-\bar{\varphi}\right)} & \bar{\varphi}=\tan ^{-1}\left(\frac{q}{k_{x}}\right) \\
0 \leq x \leq d ; & a=\sqrt{\frac{2}{1 / p+d+1 / q}} \\
a \frac{\cos (\bar{\varphi})}{\cos \left(k_{x} d-\bar{\varphi}\right)} e^{q x} & \times \cos \left(k_{x} d-\bar{\varphi}\right) \\
x \leq 0 &
\end{array}\right.
$$

where $p, q$ and $k_{x}$ are the transverse propagation constants in the cladding, in the core and in the substrate respectively. We observe that the TE modal profiles $\Psi^{\prime}$ are obtained from (4) by substituting $k_{x}^{\prime}$ to $k_{x}, p^{\prime}$ to $p, q^{\prime}$ to $q, D$ to $d, a^{\prime}$ to $a$, and $b^{\prime}$ to $b$. The wave-amplitude transmission matrix (ABCD) is used for the unit cell of Figure 1(b), in particular:

$$
\begin{aligned}
\left(\begin{array}{ll}
A & B \\
C & D
\end{array}\right)_{u}= & \left(\begin{array}{lc}
\cos \left(\theta^{\prime}\right) & i Z_{0}^{\prime} \sin \left(\theta^{\prime}\right) \\
i \sin \left(\theta^{\prime}\right) / Z_{0}^{\prime} & \cos \left(\theta^{\prime}\right)
\end{array}\right) \cdot\left(\begin{array}{cc}
r & 0 \\
0 & 1 / r
\end{array}\right) \\
& \cdot\left(\begin{array}{ll}
1 / r & 0 \\
0 & r
\end{array}\right) \cdot\left(\begin{array}{cc}
\cos \left(\theta^{\prime}\right) & i Z_{0}^{\prime} \sin \left(\theta^{\prime}\right) \\
i \sin \left(\theta^{\prime}\right) / Z_{0}^{\prime} & \cos \left(\theta^{\prime}\right)
\end{array}\right)
\end{aligned}
$$

Equation (5) provides the modal passband region through the Floquet's condition $|A+D| / 2 \leq 1$ and the total transmittivity $\left(T=2 /\left|A_{T} B_{T} C_{T} D_{T}\right|\right)^{13-16}$ of step discontinuities cascade by considering the total transmission matrix defined as:

$$
\begin{aligned}
\left(\begin{array}{ll}
A & B \\
C & D
\end{array}\right)_{\text {TотаL }}= & \left(\begin{array}{ll}
A_{T} & B_{T} \\
C_{T} & D_{T}
\end{array}\right)=\left(\begin{array}{ll}
A & B \\
C & D
\end{array}\right)_{u} \\
& \cdot\left(\begin{array}{ll}
A & B \\
C & D
\end{array}\right)_{u} \ldots\left(\begin{array}{ll}
A & B \\
C & D
\end{array}\right)_{\text {defect }} \\
& \cdots\left(\begin{array}{ll}
A & B \\
C & D
\end{array}\right)_{u} \cdot\left(\begin{array}{ll}
A & B \\
C & D
\end{array}\right)_{u}
\end{aligned}
$$

\section{RESULTS}

As example we consider a GaAs $\left(n_{2}=3.408\right)$ discontinuous waveguide of Figure 1(a) with $n_{1}=1, n_{3}=3.1$, $d=0.24 \mu \mathrm{m}, D=0.8 \mu \mathrm{m}$. By fixing the working 

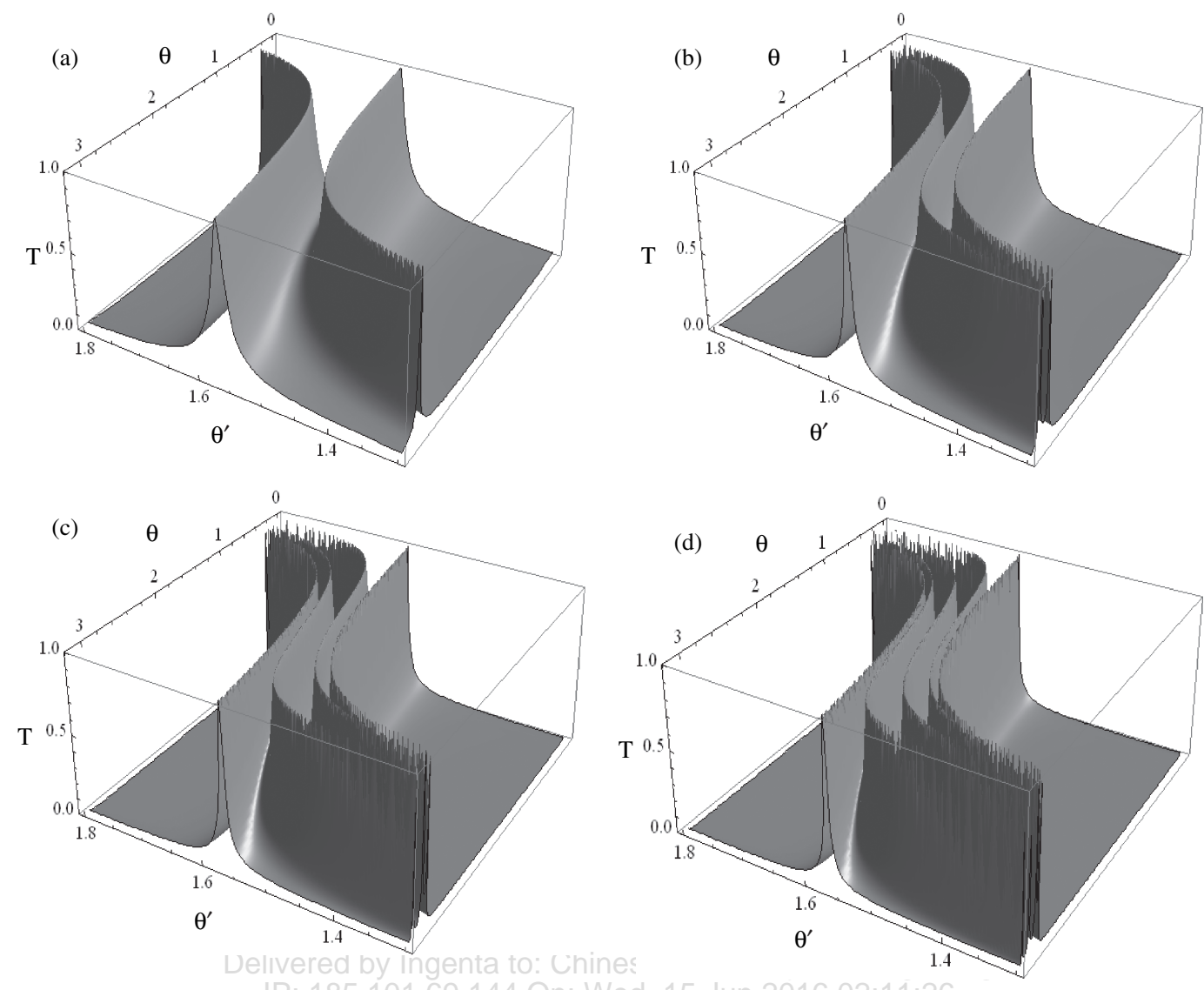

Fig. 2. Analytical transmittivity $T$ versus $\theta$ and $\theta^{\prime}$ for (a) single unit cell, (b) two unit cells, (c) three unit cells, (d) four unit cells.

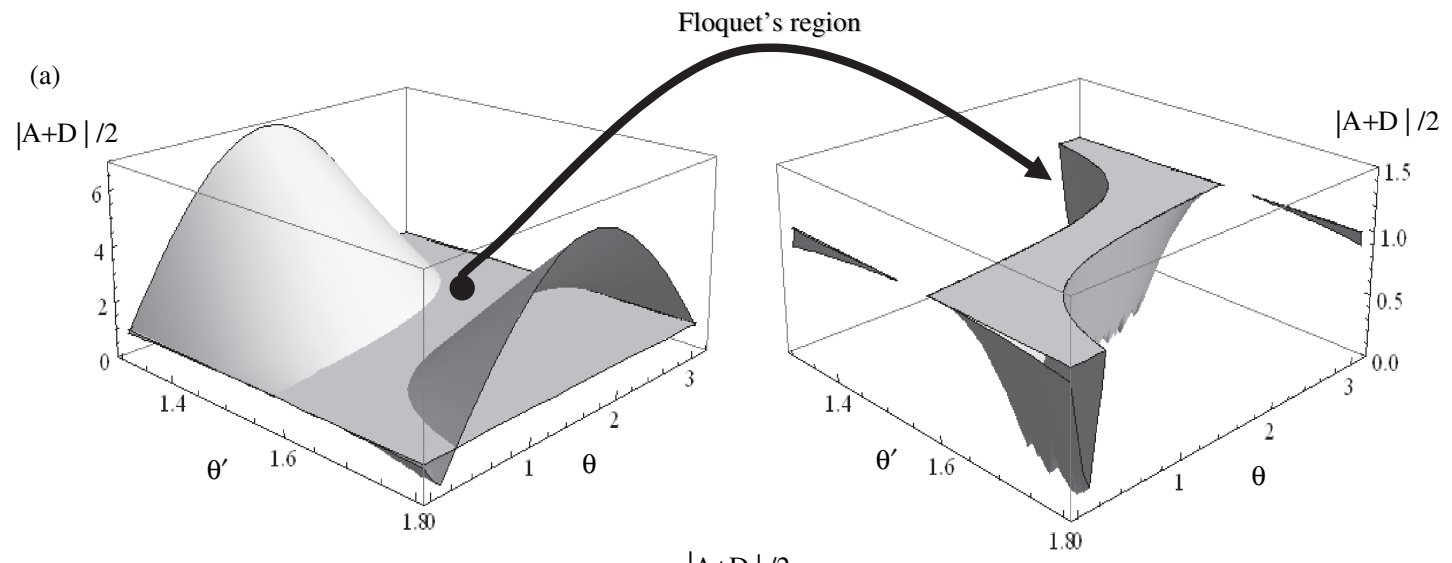

(b)

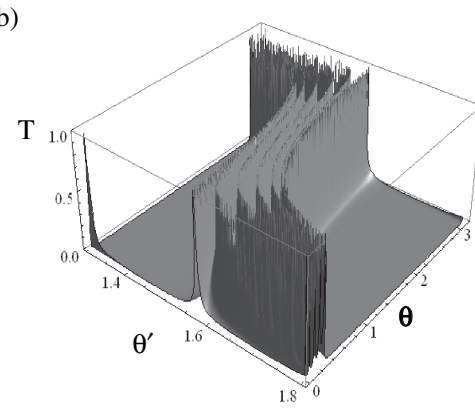

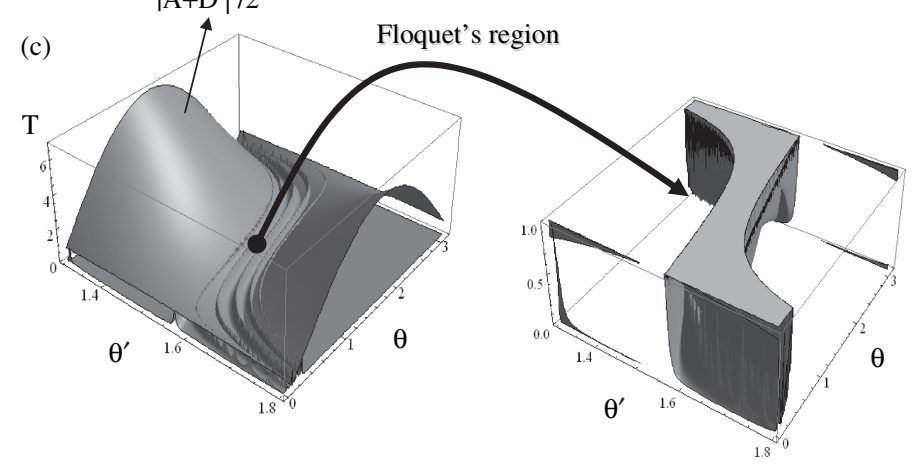

Fig. 3. (a) Floquet's region $|A+D| / 2<1$ for a single unit cell. (b) Floquet's region for five unit cells. 


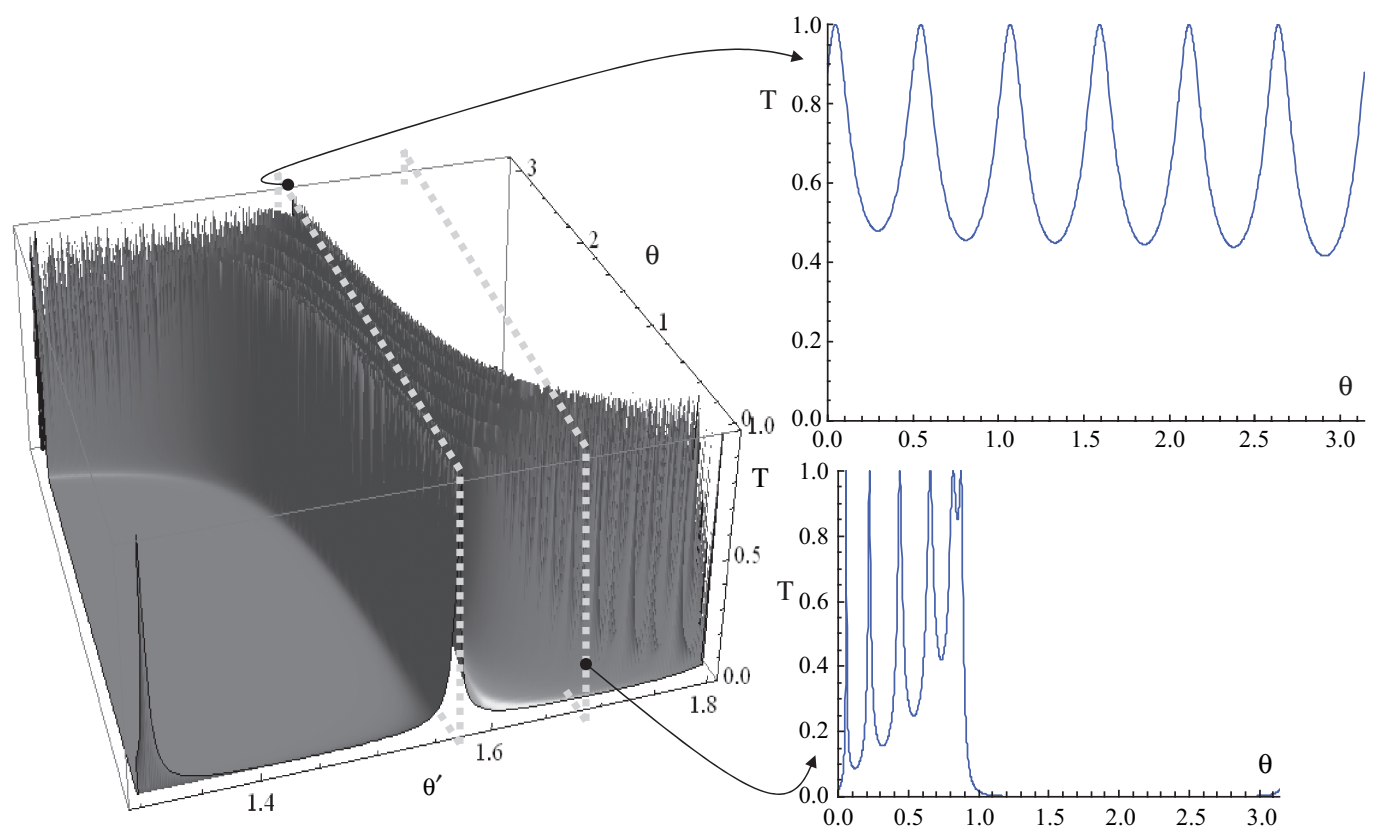

Fig. 4. Transmittivity $T$ of six unit cells versus $\theta$ and $\theta^{\prime}$ and transmittivity in the Floquet's region for $\theta^{\prime}=1.57$ and $\theta^{\prime}=1.65$.

wavelength to $\lambda_{0}=1.55 \mu \mathrm{m}$ two TE guided modes in region II and one TE guided mode in the region I will propagate. By considering the transmission line circuit referred to the fundamentals $\mathrm{TE}$ modes and by applying the effective dielectric constant method ${ }^{12,17}$ we evaluate the impedances characteristics $\mathrm{Z}_{0}^{\prime}=113.182 \Omega$ and $\mathrm{Z}_{0}=$ $120.96 \Omega$, the effective propagation constant along the $z$-propagating direction as $\beta_{z}=1.2625 * 10^{7} \mathrm{~m}^{-1}$ and $\beta_{z}^{\prime}=1.3492 * 10^{7} \mathrm{~m}^{-1}$, and finally the ratio transformation $r=5.07$ (obtained by considering all the three TE

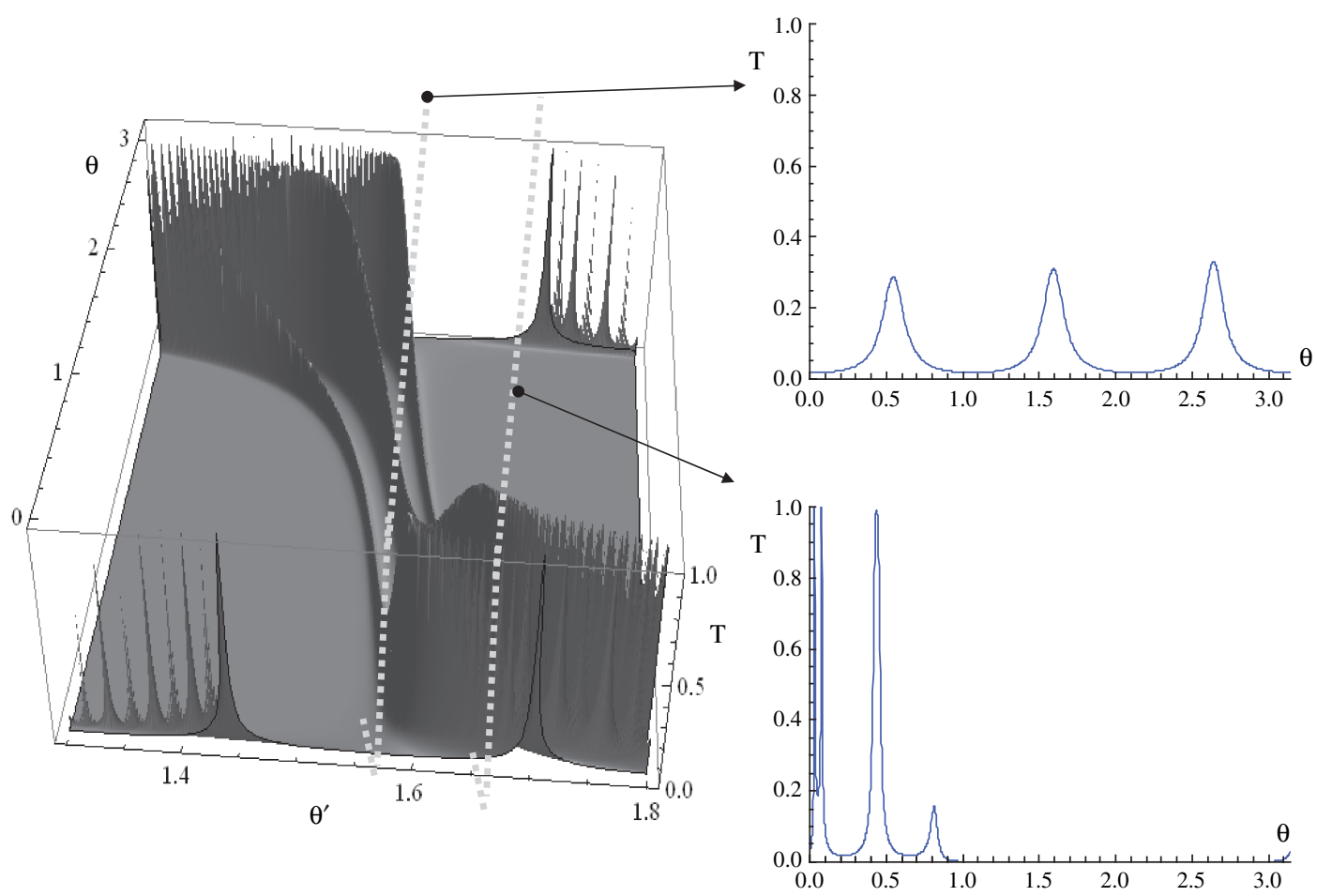

Fig. 5. Transmittivity $T$ versus $\theta$ and $\theta^{\prime}$ and transmittivity in the Floquet's region for $\theta^{\prime}=1.57$ and $\theta^{\prime}=1.65$. The configuration is 3 unit cells + air central defect +3 unit cells. 


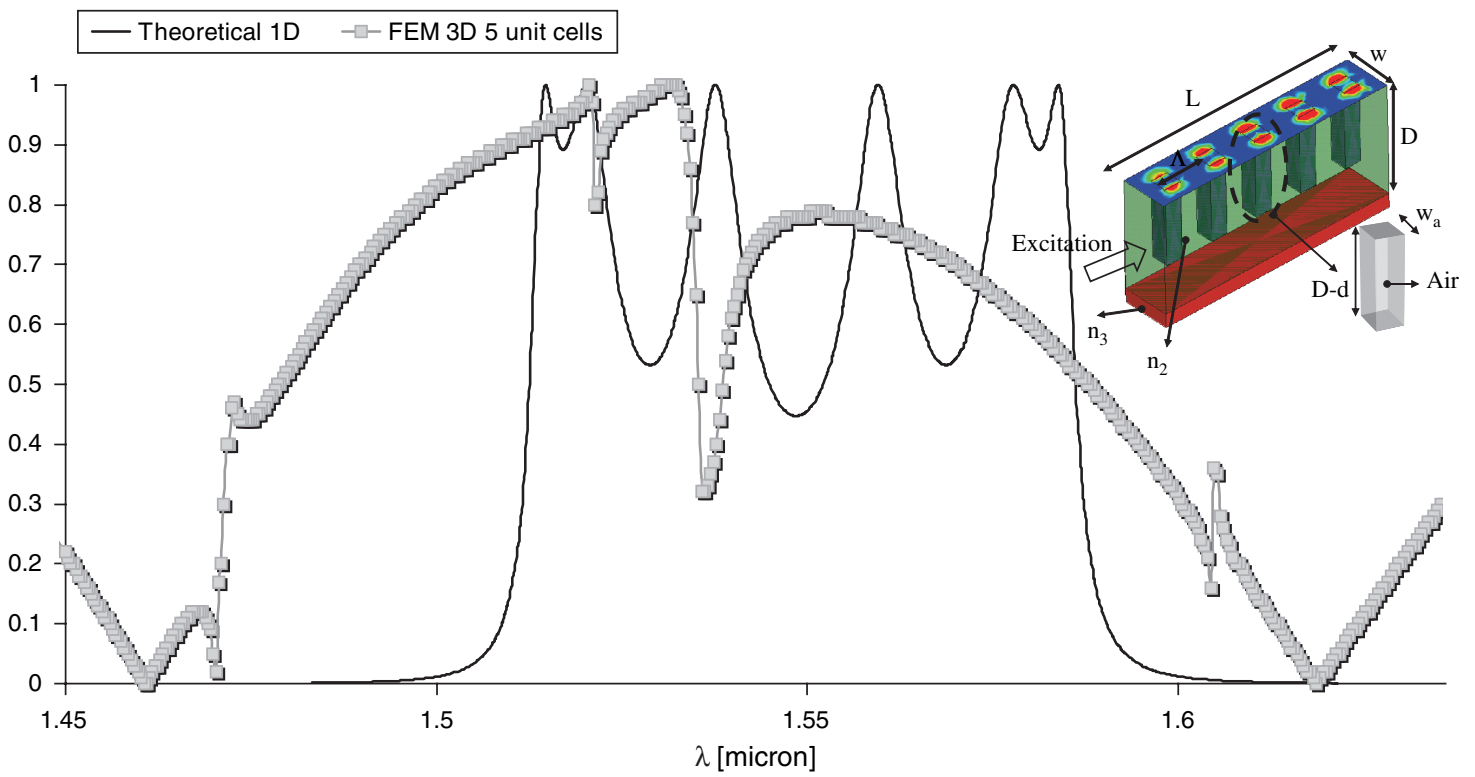

Fig. 6. Comparison between theoretical five unit cell transmittivity and 3D FEM transmittivity with transverse electric field excitation. Inset: 3D FEM resonance at $\lambda_{0}=1.55 \mu \mathrm{m}$ for a grating with $L=1.784 \mu \mathrm{m}, w=0.357 \mu \mathrm{m}, w_{a}$ (square side) $=0.1246 \mu \mathrm{m}, D=0.8 \mu \mathrm{m}, d=0.24 \mu \mathrm{m}$, $\Lambda=L_{1}+L_{2}=w_{a}+L_{2}=0.3572, n_{3}=3.1$ and $n_{2}=3.408$.

propagating modes and without considering the continuum modes). In Figures 2(a, b, c, and d) are shown the transmittivity $T$ of one, two, three, and four unit cells (discontinuity steps of the GaAs waveguide) respectively versus $\theta$ and $\theta^{\prime}$ : in this way by choosing properly values of $L_{1}$ and $L_{2}$ it is possible to obtain a high transmittivity at the preferred working wavelength. Not all the couples of $\theta, \theta^{\prime}$ will satisfy the Floquet's condition $|A+D| / 2 \leq 1$. In Figures 3(a), (b) and (c) we show how it is possible to localize the Floquet' region for a five unit cells: the Floquet's region of the single cell is superimposed to the transmission characteristic of the periodic structure by defining the passband condition. By fixing for example $\theta^{\prime}=1.57$ and $\theta^{\prime}=1.65$ it possible to define two planes which intersect the Floquet's region and the tranmittivity response as shown in Figure 4: by considering six unit cells, a high transmittivity is obtained by choosing the $\theta$ values in correspondence of the peaks. If an air defect is considered between the six unit cells (as shown in Fig. 1(a)) the $T$ characteristic is characterized by a low intensity for $\theta^{\prime}=1.57$; a better condition can be found in this case by choosing $\theta^{\prime}=1.65$ and $\theta$ in correspondence of $T$ peaks (see Fig. 5). As practical example we evaluate the passband characteristic around $\lambda_{0}=1.55 \mu \mathrm{m}$ for five unit cells by considering $L_{1}=$ $0.2326 \mu \mathrm{m}$, and $L_{2}=0.1246 \mu \mathrm{m}$. The Figure 6 shows the comparison between the theoretical 1D and the 3D FEM model of five unit cells: as expected, in both cases the passband is centered near $\lambda_{0}=1.55 \mu \mathrm{m}$, and the FEM modeling (with transverse electric field excitation) proves also the grating resonance at the working wavelength $\lambda_{0}=1.55 \mu \mathrm{m}$.

\section{CONCLUSIONS}

In this paper we introduce an accurate analytical model that studies the resonance of a discontinuous slab waveguide at high frequencies. The analytical approach predict also the effect of a central defect region in the transmittivity response. The variational equivalent circuit permits to evaluate the grating transmittivity response with a good accuracy. The theory is also validated by modeling the structure with a standard FEM-based simulator, which confirms the resonance location. The analysis can be extended also to $2 \mathrm{D}$ and $3 \mathrm{D}$ periodic patterns.

\section{References}

1. S. Noda, A. Chutinan, and M. Imada, Nature 407, 608 (2000).

2. M. Imada, S. Noda, A. Chutinan, T. Tokuda, M. Murata, and G. Sasaki, Appl. Phys. Lett. 75, 316 (1999).

3. S. Noda, M. Yokoyama, M. Imada, A. Chutinan, and M. Mochizuki, Science 393, 1123 (2001).

4. S. Fan, P. R. Villneuve, M. Joannopoulos, and J. D. Haus, Phys. Rev. Lett. 80, 960 (1998).

5. C. Manolatou, M. J. Khan, S. Fan, P. R. Villneuve, M. Joannopoulos, and J. D. Haus, IEEE J. Quantum Electron 35, 1322 (1999).

6. M. Bayindir and E. Ozbay, Opt. Express 10, 1279 (2002).

7. M. Qiu, Electron. Lett. 40, 1279 (2004).

8. M. Qiu and B. Jaskorzynska, Appl. Phys. Lett. 83, 1074 (2003).

9. A. D’Orazio, M. De Sario, V. Petruzzelli, and F. Prudenzano, Opt. Express 11, 230 (2003).

10. M. Koshiba, IEEE J. Lightw. Technol. 19, 1970 (2001).

11. A. Sharkawy, S. Shi, and D. W. Prather, Appl. Opt. 40, 2247 (2001). 
12. T. Rozzi and M. Mongiardo, Open Electromagnetic Waveguides, IEE Electromagnetic Series 43, London (1997).

13. R. E. Collin, Foundation for Microwave Engineering, 2nd edn., McGraw-Hill, New York (1992).

14. D. M. Pozar, Microwave Engineering, 2nd edn., Wiley, New York (1998).
15. A. Massaro, L. Pierantoni, and T. Rozzi, Microw. Opt. Tech. Lett. 48, 629 (2006).

16. S. G. Mao, Y. Z. Chueh, and M. S. Wu, IEEE Microw. Wireless Comp. Lett. 17, 418 (2007).

17. L. Pierantoni, A. Massaro, and T. Rozzi, IEEE Trans. Microw. Theory Tech. 53, 1856 (2005).

Received: 1 July 2008. Accepted: 15 July 2008. 